This item was submitted to Loughborough's Research Repository by the author.

Items in Figshare are protected by copyright, with all rights reserved, unless otherwise indicated.

\title{
Population balance modeling and optimization of an integrated batch crystallizer-wet mill system for crystal size distribution control
}

PLEASE CITE THE PUBLISHED VERSION

https://doi.org/10.1021/acs.cgd.7b01331

\section{PUBLISHER}

(C) American Chemical Society

\section{VERSION}

AM (Accepted Manuscript)

\section{PUBLISHER STATEMENT}

This work is made available according to the conditions of the Creative Commons Attribution-NonCommercialNoDerivatives 4.0 International (CC BY-NC-ND 4.0) licence. Full details of this licence are available at: https://creativecommons.org/licenses/by-nc-nd/4.0/

\section{LICENCE}

CC BY-NC-ND 4.0

\section{REPOSITORY RECORD}

Szilagyi, Botond, and Zoltan Nagy. 2018. "Population Balance Modeling and Optimization of an Integrated Batch Crystallizer-wet Mill System for Crystal Size Distribution Control”. figshare. https://hdl.handle.net/2134/36085. 


\title{
Population balance modeling and optimization of an integrated batch crystallizer - wet mill system for crystal size distribution control
}

\author{
Botond Szilágyi ${ }^{*+}$, Zoltán K. Nagy ${ }^{*} \dagger^{* *}$ \\ *Department of Chemical Engineering, Loughborough University, Leichestershire, \\ Loughborough Le11 3TU, United Kingdom,
}

${ }^{\dagger}$ School of Chemical Engineering, Purdue University, West Lafayette 47907-2100, USA

Keywords: crystallization control, wet mill, crystal breakage, population balances, optimization, GPU acceleration

\begin{abstract}
In this work the modeling, simulation and optimization of an integrated batch crystallizer, wet mill system is presented. It is shown that by coupling the external wet mill to the crystallizer it is possible to increase the overall system flexibility, increase the attainable crystal size distribution (CSD) and provide a significantly better distribution shaping control, than the crystallizer alone. The population balance modelling (PBM) approach with appropriate mechanisms is applied for the description of crystal population in both the crystallizer and wet mill. This description
\end{abstract}


generates a system of partial differential-integral equations, which are solved with a high resolution finite volume method, involving calculations on parallel graphical processing unit (GPU) for improved solution time. In the batch crystallizer it is assumed that primary nucleation and crystal growth are the key mechanisms, whereas in the wet mill attrition and fragmentation of crystals occurs. The nucleation and growth rate kinetics are taken from the literature and a recently developed hydrodynamic model is employed for realistic description of wet mill operation. The simulation results revealed that the simultaneous dynamic optimization of the temperature, circulation flowrate and wet mill rotation speed improve the process flexibility and lead to considerably better CSDs that can be achieved in crystallizer only configuration. The dynamic optimization also automatically discovered an unexpected optimal integrated system operation, which combines the advantages of in-situ seed generation and optimal dynamic seeding. These two features make the system suitable to achieve a significantly higher control on the shape of the CSD than using the crystallization process only, without the need of time-consuming, tailored seed crystal generation and dynamic seed addition.

\section{Introduction}

Batch crystallization is one of the most efficient and economic separation and purification techniques available in the fine chemical and pharmaceutical industry. From the particle formation point of view it is also significant, as during the batch the crystal size distribution (CSD) can be adjusted, although within a certain domain determined by supersaturation and crystallization mechanisms that occur during the batch. In most of the cases the general objective is the production of large, uniform crystals in order to facilitate the downstream operations ${ }^{1}$. However, the production of small, uniform crystals - and sometimes multimodal, or more complex CSDs, is also of great interest since these represent a way to achieve increased specific surface area and 
bioavailability. The control of crystallization processes in many practical cases consists in controlling the product $\mathrm{CSD}^{2,3}$. Since the crystallization is governed by the simultaneously ongoing nucleation and growth, which are non-linear functions of concentration, controlling their relative rates often leads to complicated control problems.

The control of batch crystallization is challenging as the initial and final system states are far from each other and during the transition the system may be characterized by significant process nonlinearities ${ }^{4}$. Generally, model predictive control (MPC) is employed to overcome the control difficulties due to process nonlinearities while respecting various constraints ${ }^{5,6}$. The widespread application of process analytical technology (PAT) tools has also lead to the development of a series of efficient model-free feedback control approaches, such as the direct nucleation control (DNC) and supersaturation control (SSC) ${ }^{7}$, which have been successfully applied for numerous crystallization systems. The batch crystallization control, regardless of the way of implementations, in most cases relies on the manipulation of temperature profile, which influences the crystallization kinetics through the supersaturation ${ }^{8,9}$. The attainable particle size domain is always delimited by the crystallization kinetics ${ }^{10}$.

In order to increase the attainable crystal size domain, additional manipulated variables, affecting crystal size, are often implied, such as antisolvent addition ${ }^{11}$ or applying combined antisolvent-temperature variations ${ }^{12}$. Conducting the crystallization so that other mechanisms such as agglomeration and breakage also occur in addition to nucleation and growth, can also be considered for better CSD control ${ }^{13-15}$. The seed addition is a very popular startup procedure of batch crystallizers, which presents high potential in tailoring the product CSD ${ }^{16}$. Growth rate modifiers were also successfully applied to fine tune the crystal size and shape during crystallization ${ }^{17,18}$. 
If the desired target CSD cannot be obtained directly in the crystallization step, then secondary operations are needed (most often milling and/or granulation) to correct the CSD. However, this involves extra operations in the production line and, may also influence negatively the quality and purity of crystals compared to the material obtained in the crystallization. Achieving process intensification by combining crystallization and milling through a recycle stream, provides a more flexible integrated system that enables a much better control of the CSD than the crystallization alone, or in fact also better control than the sequential use of crystallization and milling. The advantages and potentials of the integrated crystallization and wet-mill systems have already been demonstrated using different configurations experimentally ${ }^{19}$ and supported by models ${ }^{20}$. The work was also successfully extended to continuous wet mill based direct nucleation control for cooling crystallization ${ }^{21}$. A simulation study has also been provided to compute the attainable crystal size distribution in a similar setup ${ }^{22}$, however no optimization studies have been performed to determine the optimal operation of the integrated system.

Population balance modelling (PBM) is the widely accepted and applied approach for the mathematical description of particulate systems ${ }^{22-24}$. PBMs, from their most basic form involving nucleation and growth only with only one crystal dimension have been extended to multidimensional cases 25 and involving additional crystallization mechanisms such as fragmentation ${ }^{26}$, agglomeration ${ }^{27}$ or crystal-solution heat transfer ${ }^{28}$ among others. From process engineering perspective the fast and robust numerical solution of the PB equations (PBEs) is one of the key problems for crystallization simulation in particular for complex systems (together with the derivation of thermodynamic and kinetic expressions) ${ }^{29}$. Numerous techniques have been addressed to PBE solution from the moment based methods ${ }^{30,31}$, through Monte Carlo simulations 32 and the method of characteristics ${ }^{33}$ to discretization based techniques ${ }^{34-36}$. To improve the real 
time applicability of full PBM based simulations, which is desired as it provides the whole CSD instead of some statistically averaged quantities, the solution time is usually reduced by various techniques from using adaptive grid ${ }^{37}$, through application of parallel ${ }^{38}$ and graphical processing unit (GPU) ${ }^{39}$ based calculations to coordinate system transformations ${ }^{40}$.

Despite of the fact that PBM modelling was successfully applied for various crystallization systems and that using a recycle stream with a wet-mill can considerably widen the attainable crystal size domain, a study for the optimization of such system has not been published yet. The first objective of this work is to develop a PBM for an integrated batch crystallizer-wet mill system, involving primary nucleation, growth and dissolution for the crystallizer and fragmentation and attrition for the wet mill. The second objective is to analyze and optimize the system through numerical experimentation. The paper provides an exemplary case study that demonstrates how process intensification by system integration and the use of model-based optimization can lead to significant process performance improvements.

\section{Model development and numerical solution approach}

Let us consider a batch crystallizer, which is operated in a recirculation loop with an external wet mill (see Figure 1).

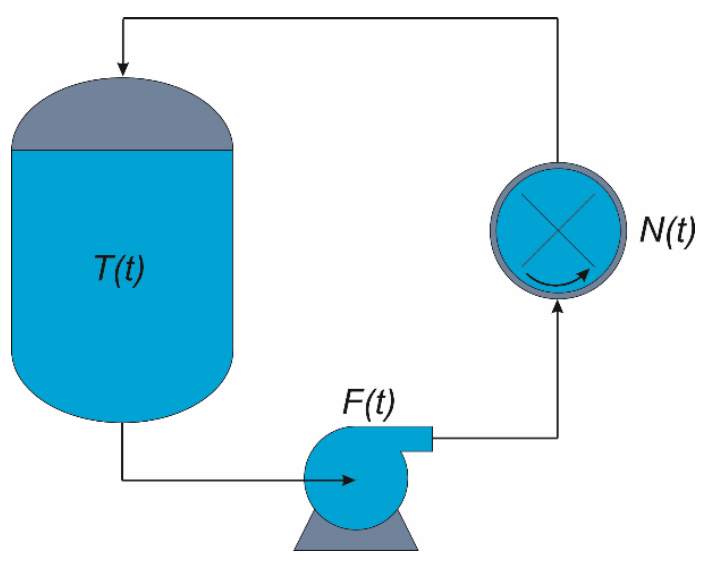


Figure 1. Schematic representation of an integrated crystallizer-wet mill system with the most important design parameters

From modeling point of view, according to Figure 1, the batch crystallizer, behaves as a continuous flow system (so-called mixed suspension mixed product removal - MSMPR), due to the constant flow in and out of the crystallizer. Similarly, the wet mill operates as a continuous milling unit. Hence, the integrated system will be modeled as two, separate sub-system which are linked by the recirculation stream.

\subsection{Modeling the crystallizer}

As simplifying assumption, ideal recirculation is considered, thus there is no time delay in the recirculation stream, and no crystallization occurs. Furthermore, in the crystallizer exclusively nucleation and crystal growth/dissolution take place, while in the wet mill only crystal breakage occurs.

For the description of particle population let us introduce the monovariate size density function $n(L, t) d L$, which gives the number of crystals within $L, L+d L$ crystal size domain per unit volume of suspension in $t$ time moment. Assuming perfect mixing in all three (micro, meso and macro) scales and homogenous suspension temperature, the PBE governing the time evolution of size density function in the crystallizer takes the form:

$\frac{\partial n_{c}(L, t)}{\partial t}+G(\sigma) \frac{\partial n_{c}(L, t)}{\partial L}=B(\sigma) \delta\left(L-L_{n}\right)+\frac{F}{V_{c}}\left[n_{w m}(L, T)-n_{c}(L, T)\right]$

With the initial and boundary conditions:

$$
\begin{aligned}
& n_{c}(L, t)=n_{c, 0}(L) \\
& \lim _{L \rightarrow \infty} n_{c}(L, t)=0
\end{aligned}
$$


In Eq.(1) the first term describes the temporal evolution of size density function, the second term stands for the crystal growth, while the right hand side is for the nucleation - assuming constant $L_{n}$ nucleon size. Subscript "wm" stands for "wet mill" while "c" for "crystallizer". $F$ is the recirculation flowrate and $V_{c}$ is the crystallizer volume. $B$ and $G$ are the nucleation and growth rates:

$$
\begin{array}{r}
B(\sigma)=\left\{\begin{array}{l}
k_{b} \sigma^{b}, \text { if } c_{c} \geq c_{s}(T) \\
0, \text { if } c_{c}<c_{s}(T)
\end{array}\right. \\
G(\sigma)=\left\{\begin{array}{l}
k_{g} \sigma^{g}, \text { if } c_{c} \geq c_{s}(T) \\
k_{d} \sigma^{d}, \text { if } c_{c}<c_{s}(T)
\end{array}\right.
\end{array}
$$

In the nucleation and growth rate expressions $k_{b}, b, k_{g}, g$ are kinetic parameters and $\sigma$ denotes the relative supersaturation:

$$
\sigma=\left\{\begin{array}{l}
\frac{c_{c}}{c_{S}(T)}-1, \text { if } c_{c} \geq c_{S}(T) \\
1-\frac{c_{c}}{c_{S}(T)}, \text { if } c_{c}<c_{S}(T)
\end{array}\right.
$$

Equations (3)-(5) are practically implemented by solving two PBMs, one with growth and nucleation for the case when the system operates in the supersaturated region and another PBM with dissolution only when the system operates in the undersaturated region.

The solubility, in mass fraction, is described as a function of temperature by the second order power law equation:

$$
c_{s}(T)=a_{0}+a_{1} T+a_{2} T^{2}
$$

The crystallizer mass balance, under the action of inlet and evacuation streams as well as of crystals nucleation and growth takes the form:

$$
\frac{d c_{c}}{d t}=-\frac{\rho_{c} k_{V}}{1-\vartheta_{C}}\left[3 G(\sigma) \int_{0}^{L_{\max }} L^{2} n_{w m}(L, t) d L+B(\sigma) L_{n}^{3}\right]+\frac{F}{V_{c}}\left(c_{w m}-c_{c}\right)
$$


where $k_{V}$ stands for the volumetric shape factor of crystals and $\rho_{c}$ is the crystal density. $\vartheta_{C}$ denote the volume fraction of crystals population, expressed by the integral term:

$$
\vartheta_{C}=k_{V} 10^{-18} \int_{0}^{\infty} L^{3} n_{c}(L, t) d L
$$

In Eq. (8) the $10^{-18}$ is the transformation factor between the $\mu m^{3} / \mathrm{m}^{3}$ dimension of CSD to $\mathrm{m}^{3} / \mathrm{m}^{3}$. As the temperature is manipulated variable, energy balance is not required for the model closure.

The set of ordinary and partial differential, as well as the accompanying kinetic and thermodynamic Eqs.(1)-(8) form the model of the batch crystallizer.

\subsection{Modeling the wet mill}

Since it is assumed that in the wet mill crystal breakage occurs exclusively, the wet mill model is a breakage PBM. Assuming perfect mixing at all scales and homogenous distribution of the mixing energy in the suspension volume, the wet-mill PBM is written as:

$$
\begin{aligned}
\frac{\partial n_{w m}(L, t)}{\partial t}= & \int_{\lambda}^{L_{\max }} b(L \mid \lambda) n_{w m}(\lambda, t) S(\lambda) d \lambda-S(L) n_{w m}(L, t) \\
& +\frac{F_{c}}{V_{w m}}\left[n_{c}(L, T)-n_{w m}(L, T)\right]
\end{aligned}
$$

The initial and boundary conditions described in Eq. (2) are valid here. The first term in the right hand side of Eq.(9) denotes the formation of crystals of size $L$ by breakage of larger particles $(\lambda)$, while the second term is the sink function of breakage. The last term takes into account the feed and evacuation streams. In Eq.(9) $S(\lambda)$ is the breakage selection function, which gives the probability that a crystal of size $\lambda$ will suffer breakage. In this work we assume the popular, size dependent selection function, involving in addition a hydrodynamic model from the literature ${ }^{13}$.

$$
S(\lambda)=\lambda^{\beta} \frac{\rho_{i m p} l_{i m p}^{2} h_{i m p} N^{3} d_{i m p}^{5}}{768 \pi}
$$


Eq.(10) correlates the impeller size and mass (density) as well as the impeller revolution speed with the breakage rate. This formulation enables the utilization of impeller revolution speed as decision variable for CSD optimization. $\beta$ is a material and system specific constant.

In Eq.(9) $b(L \mid \lambda)$ denotes the breakage function, also called as daughter distribution function. This expresses the probability that a breaking crystal of size $\lambda$ will result in a daughter crystal of size $L$. In this study we assume a combined breakage mechanism that includes both attrition and fragmentation. In this work fragmentation refers to binary breakage, that results in two crystals with comparable sizes. By attrition the specific breakage type is considered, when one of the fragments is considerably larger than the other. Hence the overall breakage rate is:

$$
b(L \mid \lambda)=b_{a}(L \mid \lambda)+b_{f}(L \mid \lambda)
$$

The attrition is modelled by a Dirac-delta function:

$$
b_{a}(L \mid \lambda)=k_{a} \delta\left(L-L_{n}\right)
$$

Eq.(12) means that the attrition produces crystals of nucleon size, i.e. crystals that contribute to the first size bin in the PBE. In this context attrition acts as a collision induced secondary nucleation. $k_{a}$ is material and system specific constant. For the description of crystal fragmentation, normal daughter CSD is assumed:

$$
b_{f}(L \mid \lambda)=k_{f} \frac{1}{\sqrt{2 \pi \sigma_{f}^{2}}} \exp \left[-\frac{(L-\lambda / 2)^{2}}{2 \sigma_{f}^{2}}\right]
$$

Eq.(13) expresses that symmetric fragmentation occurs with maximal probability but there is a certain degree of dispersion around the mean $(\lambda / 2)$, described by the dispersion factor $\sigma_{f} . k_{f}$ is material and system specific constant.

Various daughter crystals are produced under the action of fragmentation and attrition. Figure 2 illustrates an example of the overall daughter distribution function. 


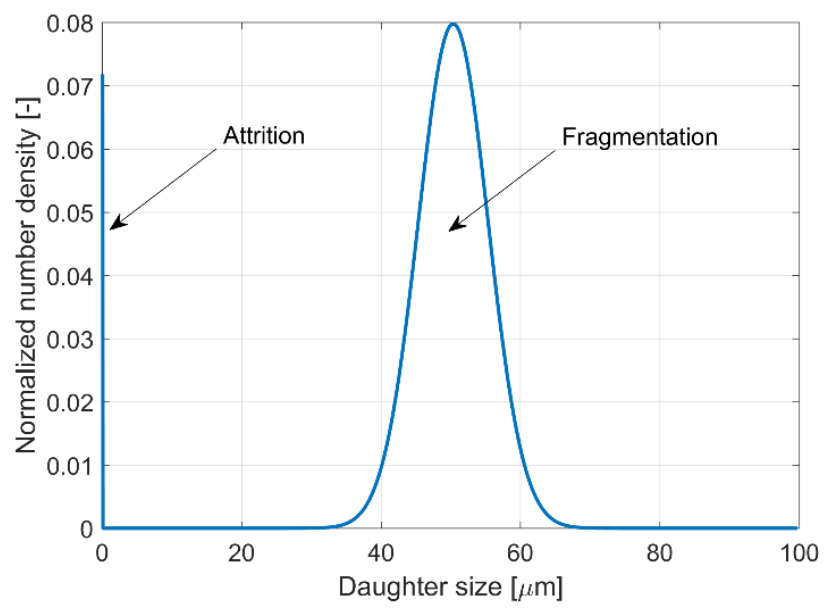

Figure 2. Representation of the overall breakage probability involving the attrition and fragmentation kernels

Despite of the assumption that in the wet mill no crystallization occurs, a mass balance is included in the wet mill model too, to take into account the dynamics and inherent inertia of the wet mill system from the point of view of concentration dynamics:

$$
\frac{d c_{w m}}{d t}=\frac{F}{V_{w m}}\left(c_{c}-c_{w m}\right)
$$

Eqs. (9)-(14) constitute the model of wet mill with attrition and fragmentation mechanisms and assuming no crystallization. These, together with Eqs. (1)-(8) form the closed model of the integrated batch crystallizer-external wet mill system.

\subsection{Finite volume solution of the model equations}

Because of their reduced computational requirements, moment based methods are generally applied to solve the breakage $\mathrm{PBE}^{41-43}$. However, recent advances in computing technologies have made possible the reduction of simulation times, leading to reasonably fast numerical solutions of the complex model equations ${ }^{44}$. Finite volume method (FVM) based solution was proposed for pure breakage PBEs ${ }^{45}$. The high resolution FVM (HR-FVM) is employed in this work to solve simultaneously the crystallizer and wet mill PBE. 
The FVM is a discretization based method, which relies on the numerical approximation of the continuous number density function, as illustrated on Figure 3.

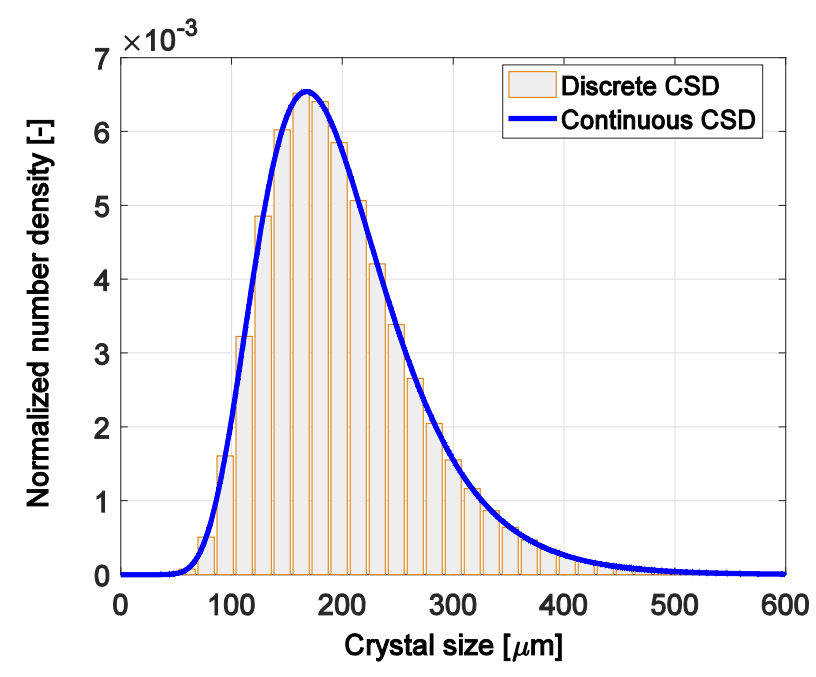

a)

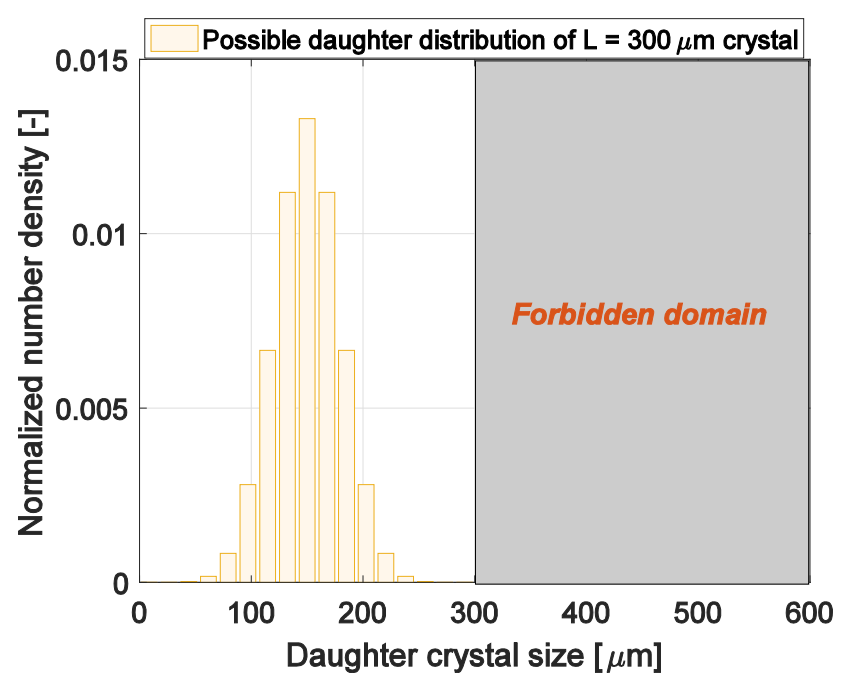

b)

Figure 3. a) Finite volume discretization of a continuous CSD; b) the illustration of fragmentation process calculation in the FVM

By definition in the FVM, the space domain is always discretized. With respect to the time coordinate, continuous and discrete descriptions are also applied. In the majority of FVM 
applications semi-discrete solution approach with continuous time formulation is employed. In contrast, if applied in custom codes, the full-discrete implementation may be more advantageous since it does not require ordinary differential equation solvers ${ }^{39}$. Hence, in this study a full-discrete implementation is used.

Let $h_{l}$ denote the size of the $l^{\text {th }}$ grid cell and $k$ the (discrete) time step size. Then, $n_{l, c}^{m}$ is the averaged approximation of the crystallizer population density:

$$
n_{l, c}^{m} \approx \frac{1}{h_{l}} \int_{(l-1) h}^{l h} n_{c}(x, m k) d x
$$

The wet mill population $\left(n_{l, w m}^{m}\right)$ is discretized in similar manner. In Eq.(15) $m$ and $l$ are integers such that $m \geq 0$ and $M \geq l \geq 1 . M$ denotes the mesh size (i.e. the number of discretization points).

The FVM computes the value of $n_{l, c}^{m}$ in the next time step $\left(n_{l, c}^{m+1}\right)$ under the action of crystal nucleation, growth and input-output streams. Then, the calculations are repeated until the final time is reached. LeVeque ${ }^{46}$ presented a FVM for such hyperbolic system, where the growth rates are evaluated at the endpoints of each grid cell. This method has been adopted in many studies in the field of crystallization. Applying the FVM on the crystallizer PBE (1) the following algebraic equation is generated:

$$
\begin{aligned}
n_{l, c}^{m+1}=n_{l, c}^{m} & -k\left(\frac{G_{l}}{h_{l}} n_{l, c}^{m}-\frac{G_{l-1}}{h_{l-1}} n_{l-1, c}^{m}\right) \\
& -\left[\frac{k G_{l}}{2 h_{l}}\left(1-\frac{k G_{l}}{h}\right)\left(n_{l+1, c}^{m}-n_{l, c}^{m}\right) \phi_{l}\right. \\
& \left.-\frac{k G_{l-1}}{2 h_{l-1}}\left(1-\frac{k G_{l-1}}{h_{l-1}}\right)\left(n_{l, c}^{m}-n_{l-1, c}^{m}\right) \phi_{l-1}\right]+\epsilon \frac{B}{h_{1}} \\
& +k \frac{F}{V_{c}}\left(n_{l, w m}^{m}-n_{l, c}^{m}\right)
\end{aligned}
$$

The first part in the right hand side is the actual particle number in cell $l$, the second is the first order term which describes the growth and the third is the second order growth term. $\epsilon$ in the nucleation term is a binary variable with values $\{0,1\}$ defined as $\epsilon=1$ if $h=1$ and it is 0 
otherwise. This means that the nucleation exists at the lower bound of the crystal size domain only. The solution of Eq.(16) is straightforward for growth problems. For modelling the dissolution case, the finite volume scheme practically has been inverted: during the crystal growth direction of flux was from liquid to solid phase, where the nucleation is a boundary condition of the left boundary, whereas for dissolution the flux direction was from solid to liquid, in which case the right boundary is described by Eq.(2).

According to Eq.(16), the calculation of $n_{l}^{m+1}$ requires the knowledge of $n_{l}^{m}$ (the distribution in the current time step, that can be either the result of a previous FVM iteration or the initial condition), the time step size, growth-and nucleation rates and the $\phi_{l}$ function. In the equation, $\phi_{l}$ denotes the flux limiter function, which depends on the degree of smoothness of the distribution. The smoothness is expressed as the ratio of two consecutive gradients:

$$
\theta_{l}=\frac{n_{l}^{m}-n_{l-1}^{m}}{n_{l+1}^{m}-n_{l}^{m}}
$$

The flux limiter function has the role to switch adaptively between the first and second order schemes in the sharp regions, where the second order formulation tends to produce numerical oscillations. The Van Leer flux limiter has been successfully applied in the simulation of PBEs and provides full second order accuracy for $1 \mathrm{D}$ problems ${ }^{35}$. This flux limiter has the form:

$$
\phi\left(\theta_{l}\right)=\frac{\left|\theta_{l}\right|+\theta_{l}}{1+\left|\theta_{l}\right|}
$$

Finally, the flux limiter function provides the HR feature for the FVM. The time step $k$ is calculated based on the Courant, or also called CFL (Courant-Friedrichs-Lewy) number:

$$
k=C \max \left(\frac{h_{l}}{G_{l}}\right)
$$


In Eq.(19) $C$ denotes the Courant number. For FVM the $C \leq 1$ inequality ensures the solver stability. According to the criterion, the time step size is calculated in the function of growth rate to avoid the crystal grow through more than one size bin.

Related to the simulation of breakage processes, the two most important quantities are the crystal number and crystal mass. The crystal mass must be conserved during the process while the crystal number must increase in agreement with the breakage frequency. In this work the calculated daughter CSD is normalized, then re-scaled based on its third moment to match the third moment of the breaking crystal. This treatment ensures mass conservation, regardless of mesh quality. The mesh must be validated for crystal number simulation.

The daughter distribution discretization, based on the FVM approximation, takes the form:

$$
\tilde{n}_{l \mid \kappa}^{m+1} \approx k \int_{(l-1) h}^{l h} b\left(x \mid d_{\kappa}\right) n_{w m}\left(d_{\kappa}, m k\right) d x
$$

In Eq. (20) $d_{\kappa}$ denotes the mean size of the $\kappa^{\text {th }}$ cell, $\tilde{n}_{l \mid \kappa}^{m+1}$ is the number of daughter crystals of size $L$ (corresponding to the $l^{\text {th }}$ grid cell), born from the breakage of a crystal of size $\lambda$ (corresponding to $\kappa^{\text {th }}$ grid). This simple equation, however, will require adjustment since it does not take into consideration the grid size $\left(h_{l}\right)$. This will be done by the following two equations. By using the selection function, the third moment of crystals fraction of size $\lambda$, which suffers breakage can be expressed easily:

$$
\mu_{3, \kappa}^{m}=k S\left(d_{\kappa}\right) h_{\kappa} d_{\kappa}^{3}
$$

By combining Eqs. (20)-(21), the mass conserving daughter crystals population can be expressed as:

$$
n_{l \mid \kappa}^{m+1}=\tilde{n}_{l \mid \kappa}^{m+1} \frac{h_{l} d_{l}^{3}}{\mu_{3, \kappa}^{m}}
$$


In order to calculate the overall daughter crystals population, the individual daughter populations are summed up:

$$
n_{l, \text { dau }}^{m+1}=\sum_{i=1}^{M} n_{l \mid i}^{m+1}
$$

With these notations the wet-mill FVM takes the form:

$$
n_{l, w m}^{m+1}=n_{l, d a u}^{m+1}-\mu_{3, \kappa}^{m}+k \frac{F}{V_{w m}}\left(n_{l, c}^{m}-n_{l, w m}^{m}\right)
$$

The $k$ time step size used in the wet-mill calculations is the same to the one calculated by Eq.(19). After the crystallizer and wet mill PB, the discretized mass balances are required. The crystallizer mass balance is:

$$
\begin{aligned}
c_{c}^{m+1}=c_{c}^{m} & -\varphi \frac{3 k_{v} \rho_{C}}{1-\vartheta_{C}}\left(G(\sigma) \sum_{l=1}^{N} h_{i} l^{2} n_{l, c}^{m}+B(\sigma) h_{1} l_{1}^{3}\right) \\
& +k \frac{F}{V_{c}}\left(c_{w m}^{m}-c_{c}^{m}\right)
\end{aligned}
$$

In Eq.(25) $\varphi=1$ if the solution is saturated and it is -1 otherwise. With this constant the algorithm takes into account that during the crystal growth the solute concentration decreases and it increases during the dissolution. The wet mill mass balance takes the form:

$$
c \underset{w m}{m+1}=c_{w m}^{m}+k \frac{F}{V_{w m}}\left(c_{c}^{m}-c_{w m}^{m}\right)
$$

The nonlinear algebraic equation system model Eqs.(15)-(26) is the discretized form of the integrated crystallizer-external wet mill model.

\subsection{Numerical details and grid independence study}

The HR-FVM simulation is expected to be time consuming due to the increased number of model equations (Eq.(16) and (24)). To improve the simulation time, efficient computer implementation is required, involving fast programming languages, such as $\mathrm{C}$ and parallel 
computing. GPUs are often used to accelerate scientific calculations due to their inherent and efficient parallel hardware architecture. As the generated equation system presents high parallelization potential (keeping in mind that $l$ in Eq.(16) denotes the cell number, thus it is clear that similar algebraic equations are solved for each cell), in addition to the serial C code a GPU accelerated solver written in CUDA C language has also been developed. Both codes are compiled to .mex functions and are called directly from the Matlab software environment. In the GPU accelerated solver the serial operations (mass balance, supersaturation, nucleation and growth rate calculations as well as the adaptive time stepping) are executed on the CPU, whereas the parallelizable operations (finite volume calculations and integrals) are executed on the GPU. Hence, the most efficient implementation involves a hybrid calculation, utilizing simultaneously and optimally the calculation resources of the computer ${ }^{39}$. It must be highlighted that because of the substantially different algorithmic implementation and compilation of serial (CPU) and hybrid $(\mathrm{CPU}+\mathrm{GPU})$ solvers, for crude meshes the results may slightly differ. However, for a valid, sufficiently fine mesh the results must be overlapping.

The kinetic and process data used in the process simulations are listed in Table 1.

Table 1. Process and kinetic constants used in the simulations

\begin{tabular}{cll}
\hline Parameter & Name [U.M.] & Value \\
\hline$V_{c}$ & Crystallizer volume $\left[\mathrm{m}^{3}\right]$ & $1.0 \cdot 10^{-3}$ \\
$V_{w m}$ & Wet mill volume $\left[\mathrm{m}^{3}\right]$ & $1.5 \cdot 10^{-4}$ \\
$k_{V}$ & Volumetric shape factor $[-]$ & 0.32 \\
$\rho_{C}$ & Crystal density $\left[\mathrm{kg} / \mathrm{m}^{3}\right]$ & 1412 \\
$a_{0}$ & Solubility parameter $[\mathrm{kg} / \mathrm{kg}]$ & $5.48 \cdot 10^{-3}$ \\
$a_{1}$ & Solubility parameter $\left[\mathrm{kg} / \mathrm{kg}{ }^{\circ} \mathrm{C}\right]$ & $-1.93 \cdot 10^{-4}$ \\
$a_{2}$ & Solubility parameter $\left[\mathrm{kg} / \mathrm{kg}{ }^{\circ} \mathrm{C}^{2}\right]$ & $7.09 \cdot 10^{-6}$ \\
\hline
\end{tabular}




\begin{tabular}{|c|c|c|}
\hline$k_{b}$ & Nucleation rate constant $\left[\# / \mathrm{m}^{3} \mathrm{~s}\right]$ & $1.39 \cdot 10^{5}$ \\
\hline$b$ & Nucleation rate exponent $[-]$ & 2.62 \\
\hline$k_{g}$ & Growth rate constant $[\mathrm{m} / \mathrm{s}]$ & 0.337 \\
\hline$g$ & Growth rate exponent $[-]$ & 1 \\
\hline$k_{d}$ & Dissolution rate constant $[\mathrm{m} / \mathrm{s}]$ & 0.563 \\
\hline$d$ & Dissolution rate exponent [-] & 1 \\
\hline$\rho_{i m p}$ & Impeller density $\left[\mathrm{kg} / \mathrm{m}^{3}\right]$ & 7850 \\
\hline$h_{i m p}$ & Impeller thickness $[\mathrm{m}]$ & $2 \cdot 10^{-3}$ \\
\hline$l_{i m p}$ & Impeller height $\left[\mathrm{kg} / \mathrm{m}^{3}\right]$ & $50 \cdot 10^{-3}$ \\
\hline$d_{i m p}$ & Impeller diameter $[\mathrm{m}]$ & $60 \cdot 10^{-3}$ \\
\hline$\beta$ & Selection function exponent $[-]$ & 1 \\
\hline$k_{a}$ & Attrition rate constant $\left[\# / \mathrm{m}^{3} \mathrm{~s}\right]$ & 200 \\
\hline$k_{f}$ & Fragmentation rate constant $\left[\# / \mathrm{m}^{3} \mathrm{~s}\right]$ & 80 \\
\hline$\sigma_{f}$ & $\begin{array}{l}\text { Standard deviation of daughter } \\
\text { distribution }[-]\end{array}$ & $\lambda / 100$ \\
\hline
\end{tabular}

The material properties and crystallization kinetic data of Table 1 refers to the ortho-amino benzoic acid (oABA) published in literature ${ }^{47}$. The breakage constants were chosen to illustrate an estimated effect of breakage on the CSD.

The numerical accuracy of a discretization based solver, like the HR-FVM, may depend on the mesh quality. The estimation of numerical errors is routinely performed in the field of computational fluid dynamics simulations ${ }^{48}$, however, it got less attention in the FVM based PBE solution. In order to validate the mesh, simulations are carried out using both the CPU and CPU+GPU solvers with different space (mesh size) and time (CFL) discretization. In these 
simulations the $1200 \mu \mathrm{m}$ maximal crystal size was chosen based on the results of a linear cooling simulation - without recirculation - where the maximal size was $<1000 \mu \mathrm{m}$.

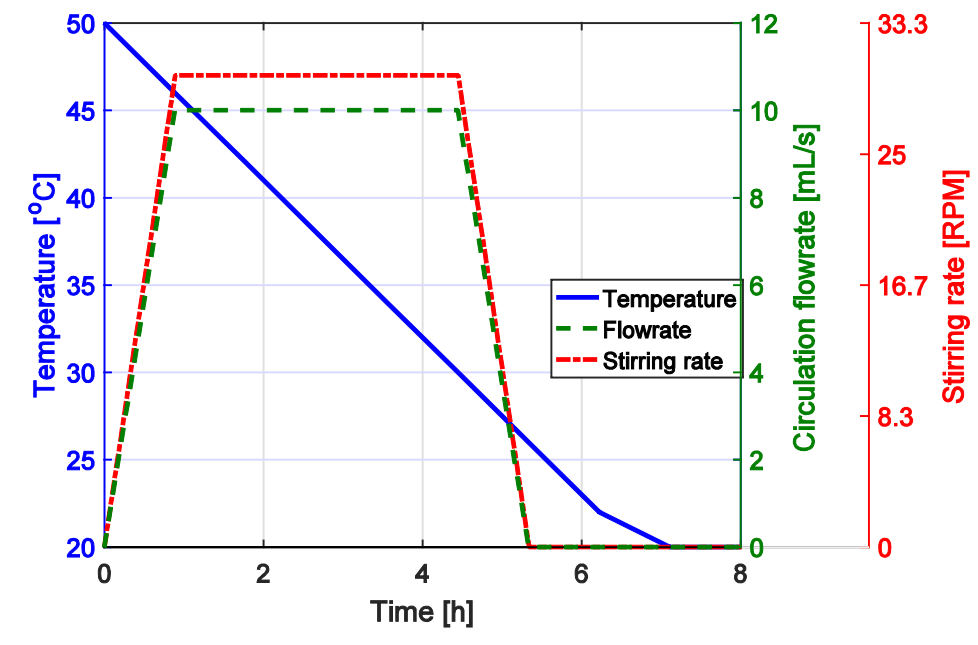

a)

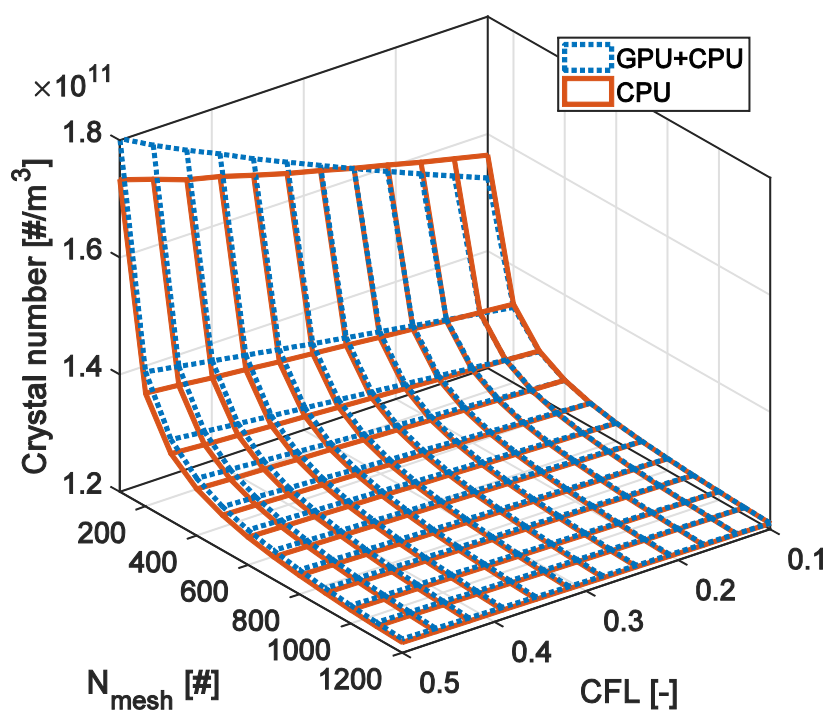

b) 


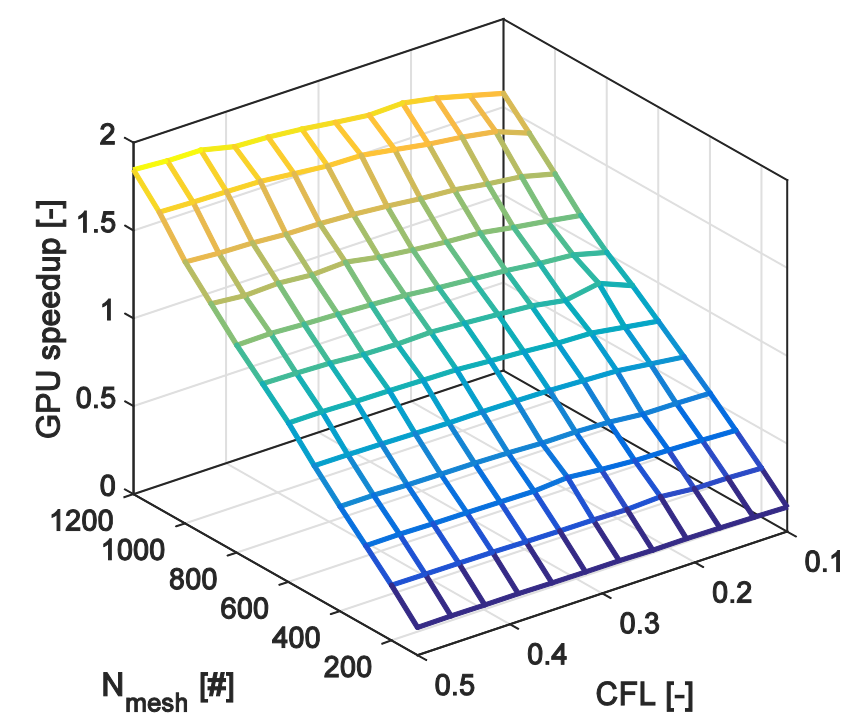

c)

Figure 4. a) Flowrate, temperature and stirring rate profiles used in the mesh validation experiments; b) Crystal number predicted by the GPU accelerated and the serial solver; c) Degree of acceleration of GPU over the serial solver

According to the Figure 4 it is obvious that using too fine mesh does not bring additional accuracy but leads to excessive simulations times. Three domains can be distinguished with respect to the solution accuracy and the optimal utilization of computational resources (see in Table 2).

Table 2. Potential discretization domains for numerical simulations

\begin{tabular}{llll}
\hline Accuracy & CFL & Mesh size & Computing platform \\
\hline High & CFL $<0.3$ & $\mathrm{~N}>1000$ & CPU + GPU \\
Moderate & $\mathrm{CFL}>0.3$ & $\mathrm{~N}>500$ & \\
& $\mathrm{CFL}<0.5$ & $\mathrm{~N}<1000$ & CPU + GPU; CPU only \\
Low & $\mathrm{CFL}>0.5$ & $\mathrm{~N}<500$ & CPU only \\
\hline
\end{tabular}


It has been showed that even approximate HR-FVM solutions can be successfully used in nonlinear MPC, if coupled with robust state estimator ${ }^{49}$. Although, for design and optimization purposes the application of highly accurate models is required.

\section{Optimization results and discussions}

The objective of this section is to analyze the dynamic optimization possibilities of the integrated crystallizer-wet mill system for the production of a desired CSD. Being a dynamically operated system, the decision variables are the profiles of crystallizer temperature, the circulation flowrate and the wet mill stirring rate. The optimization is subject to various constraints, listed in Table 3 . Table 3. Constraints of the dynamic crystallizer-wet mill optimization

\begin{tabular}{cll}
\hline Notation & Meaning [U.M.] & Value \\
\hline$T_{\text {max }}$ & Maximal temperature $\left[{ }^{\circ} \mathrm{C}\right]$ & 60 \\
$T_{\text {min }}$ & Minimal temperature $\left[{ }^{\circ} \mathrm{C}\right]$ & 20 \\
$T_{\text {max } \text { end }}$ & Maximal final temperature $\left[{ }^{\circ} \mathrm{C}\right]$ & 46 \\
$N_{\text {max }}$ & Maximal wet mill RPM [RPS] & 80 \\
$N_{\text {min }}$ & Minimal wet mill RPM [RPS] & 0 \\
$F_{\text {max }}$ & Maximal recirculation flowrate $[\mathrm{mL} / \mathrm{s}]$ & 15 \\
$F_{\text {min }}$ & Minimal recirculation flowrate $[\mathrm{mL} / \mathrm{s}]$ & 0 \\
$c r_{\text {max }}$ & Maximal cooling rate $\left[{ }^{\circ} \mathrm{C} / \mathrm{min}\right]$ & 0.5 \\
$c r_{\text {min }}$ & Minimal cooling rate $\left[{ }^{\circ} \mathrm{C} / \mathrm{min}\right]$ & -0.5 \\
$\Delta F_{\text {min }}$ & Maximal flowrate variation $\left[\mathrm{mL} / \mathrm{s}{ }^{2}\right]$ & 1 \\
$\Delta N_{\text {min }}$ & Maximal stirring rate variation $[\mathrm{RMS} / \mathrm{s}]$ & 1 \\
\hline
\end{tabular}


Note that the optimization constraints of Table 3 may be of technical or economical nature, but rationally selected constraints may also accelerate the convergence of optimization by reducing the search-space.

Denoting the profiles of temperature, flowrate and stirring by $[T, F, N]$, a sum-squared error (SSE) based goal function can be formulated, taking into account multiple objectives:

$$
\begin{aligned}
\operatorname{SSE}(T, F, N)= & \int_{0}^{L_{\max }}\left[\frac{\left|n_{T}\left(L, t_{\text {end }}\right)-n\left(L, t_{\text {end }}\right)\right|+\varepsilon}{n_{T}\left(L, t_{\text {end }}\right)+\varepsilon}\right]^{2} d L \\
& +\int_{0}^{t_{\text {end }}}\left[w_{1}\left(\frac{d T}{d t}\right)^{2}+w_{2}\left(\frac{d F}{d t}\right)^{2}+w_{3}\left(\frac{d N}{d t}\right)^{2}\right] d t+\int_{0}^{t_{\text {end }}}\left[w_{4} F^{2}+w_{5} N^{2}\right] d t \\
& +w_{6}\left(T_{\text {fin }}\right)^{2}
\end{aligned}
$$

The first term of the objective function minimizes the difference between the target and predicted final CSD, where $\varepsilon$ is a small number included to avoid the division by zero. The second term ensures smooth profile variations; the third term forces the algorithm to minimal pump and wet mill utilization. Finally, the last term is included for economic reasons to maximize yield, since the final temperature is correlated directly with the yield. Yield is not included as a hard constraint in this optimization since the main aim here is to see if a target CSD can be achieved in a fixed batch time. Yield and CSD maybe conflicting objectives when the duration of the batch is fixed. The weighting factors used in the optimization were chosen based on the results of preliminary optimizations and, finally, were set to $w_{1}=4 \times 10^{6} ; w_{2}=2 \times 10^{6} ; w_{3}=1000 ; w_{4}=4 ; w_{5}=0.2$ and $w_{6}=$ 0.06. In all optimizations 30-minute equilibration time was applied, which means that the last temperature was kept constant to desupersaturate the solution.

In order to reduce the optimization time, a three level optimization approach was carried out, increasing gradually the number of discretization points in the temperature, flowrate and stirring 
rate profiles. At the same time, the solution accuracy was increased gradually as well (see Table 4).

Table 4. Three-level sequential optimization procedure

\begin{tabular}{ccc}
\hline Level & Number of elements/profile & Solution accuracy (Table 2) \\
\hline 1 & 10 & Low \\
2 & 25 & Moderate \\
3 & 35 & High \\
\hline
\end{tabular}

According to the Table 4, the third level optimization is highly accurate and it involves the most decision variables. Considering the increased computational burden, it is particularly important the existence of a good starting point. This is provided by the Level 2 optimization, for which, in similar manner, the Level 1 optimization provides the initial point. The simulations revealed that this pseudo warm-start is considerably faster than by running a sole optimization with the high accuracy solution (Level 3 only). All optimizations are carried out using the interior-point method by calling the fmincon function of Matlab.

In optimization studies three cases are evaluated: the production of (i) large (400 $\mu \mathrm{m}$ mean size), (ii) small (200 $\mu \mathrm{m}$ mean size) and (iii) a bimodal CSD. To highlight the importance of the wet mill/recirculation stream, the optimization of the crystallizer only (without wet mill/recirculation) is also carried out and presented.

In the first case study a log-normally distributed population is set as the target CSD with $400 \mu \mathrm{m}$ mean size and $100 \mu \mathrm{m}$ dispersion. According to the Figure 5b, the target CSD was not even approximated if only the crystallizer was considered, however, the product CSD produced by the integrated system overlaps almost completely with the target CSD. This case study illustrates how by using dynamic process optimization it is possible to discover a novel operating strategy, which 
has not been proposed before in the studies dealing with similar setups. The optimal operation can be divided to the following stages:

Seed generation stage in crystallizer: the crystallizer is cooled to generate nucleation and crystal growth. During this stage, the pump and wet mill are both turned off.

Seed transfer to wet mill and dissolution of excess crystals: when the crystals appear in the crystallizer, the pump is turned on to transport some of the crystals to the wet mill. The crystallizer temperature is increased to start dissolving excess crystals.

Generation of optimized seed CSD in the wet-mill: the pump is turned off, which decouples the wet mill from the crystallizer. During this time a complete dissolution occurs in the crystallizer followed by a temperature decrease to a supersaturated stage, while the wet mill is operated in high rotation speed to generate small seed crystals. This seed CSD is smaller and narrower than what could be achieved in the crystallizer only via primary nucleation.

Controlled crystallization with dynamic seeding using the optimal seed CSD: in the last stage, after seed is transferred into the crystallizer the wet mill is turned off and an optimal temperature profile that follows a typical parabolic temperature profile to minimize secondary nucleation, is applied in the crystallizer. The pump is operated in controlled manner, which, acts as a dynamic optimal seeder of the crystallizer. 


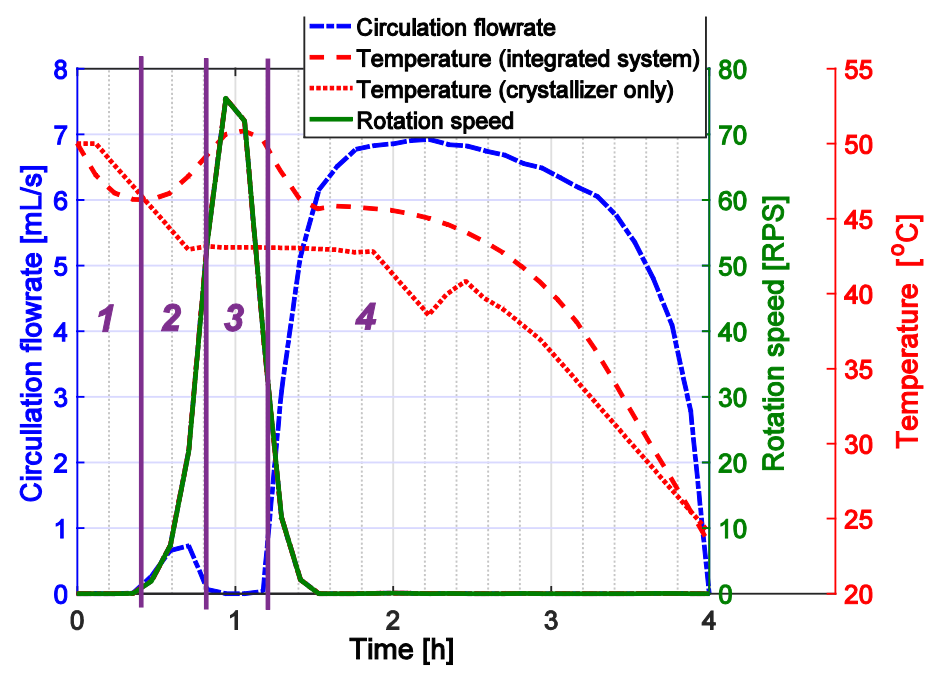

a)

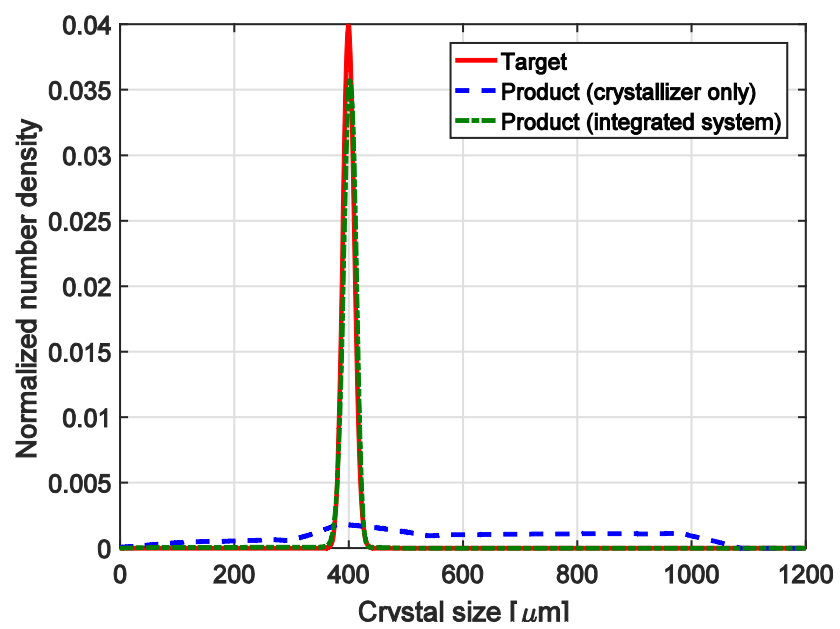

b)

Figure 5. First optimization study - target CSD with $400 \mu \mathrm{m}$ mean size: a) optimal trajectories of temperature, stirring rate and circulation flowrate; and b) the target CSD as well as the achieved product CSDs of the two systems (with and without wet mill)

Very similar system behavior can be observed in Figure 6, when a target CSD of $200 \mu \mathrm{m}$ mean and $70 \mu \mathrm{m}$ dispersion was used. The crystallizer-only setup cannot produce the desired CSD, in contrast, a very wide crystal population can only be achieved, whereas in the integrated system 
operation the in-situ seed-generation and optimal dynamic seeding is implemented with very good product CSD.

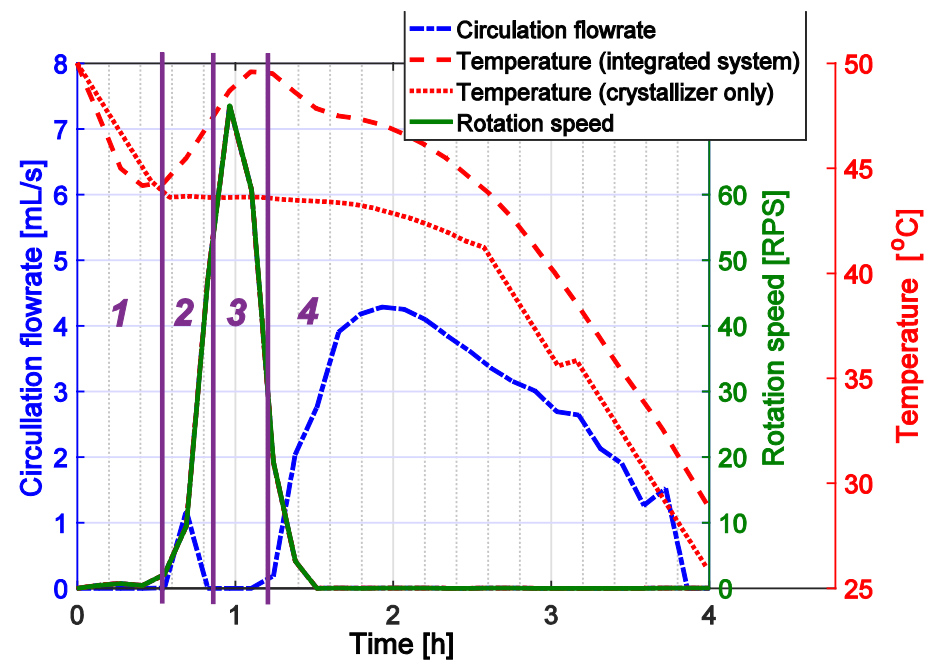

a)

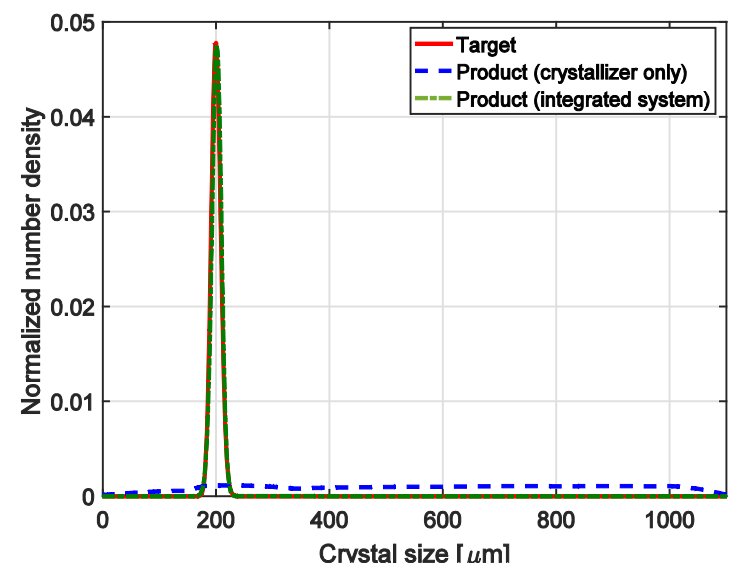

b)

Figure 6. Second optimization study - target CSD with $200 \mu \mathrm{m}$ mean size: a) optimal trajectories of temperature, stirring rate and circulation flowrate; and b) the target CSD as well as the achieved product CSDs of the two systems (with and without wet mill). 
In the last case study, a bimodal target CSD was considered. This might be required for controlled release purposes through controlled dissolution ${ }^{50}$. Since in this target CSD the crystal sizes are considerably lower than in the previous case studies, here a 1-hour batch time will be applied, which also serves as a good test for the possibility of batch time reduction/optimization. In this situation a more complicated system behavior is observed. The pump in principle is operated in similar manner as in the previous case studies: first, it transports some crystals to the wet mill, then it is stopped and finally it transports back the generated seeds, with optimized CSD via milling, to the crystallizer. However, in contrast to the previous case studies, the lower CSD peak is generated by a second, powerful milling stage, during which, the pump is not turned off. This is due to the short batch time. To avoid the growth of seeds, which are transported to the crystallizer during the simultaneous wet-mill and pump operation, the crystallizer is heated to slowly but continuously dissolve the fines. When the wet-mill is turned off, the second cooling stage begins, which generates the growth of existing crystals, which will form the large crystal fraction, and the seeds, coming from the wet mill - to create the smaller peak of the target CSD.

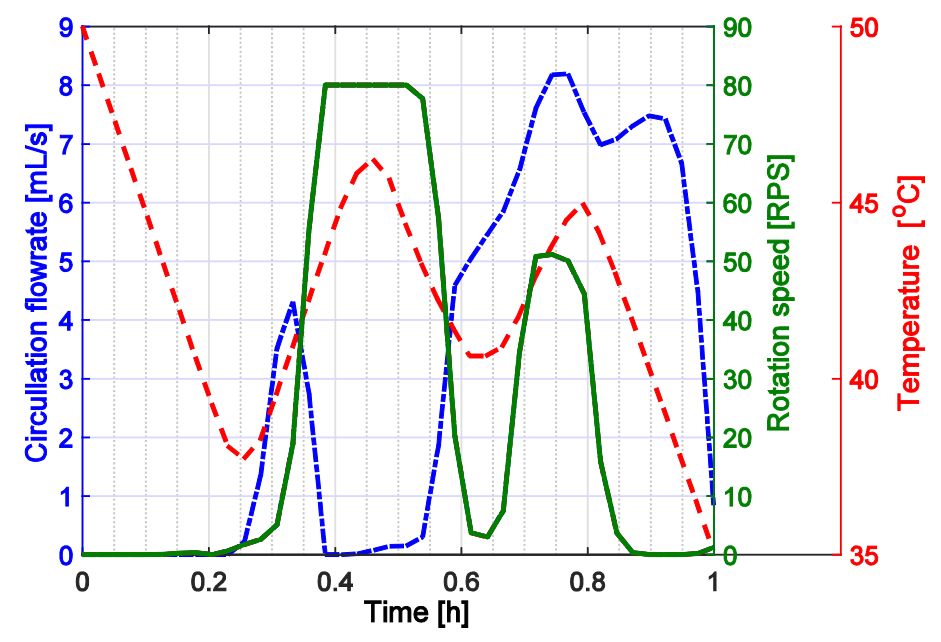

a) 


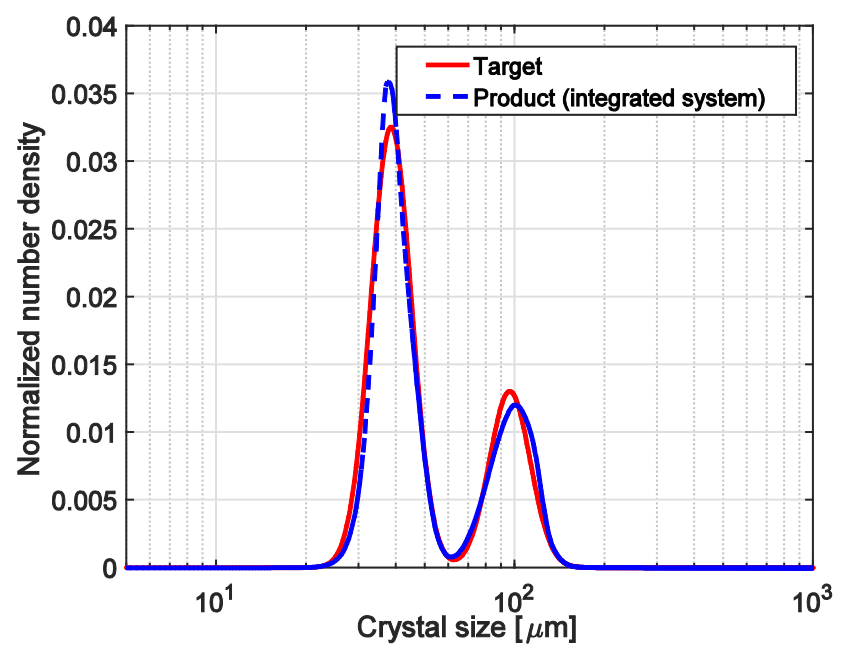

b)

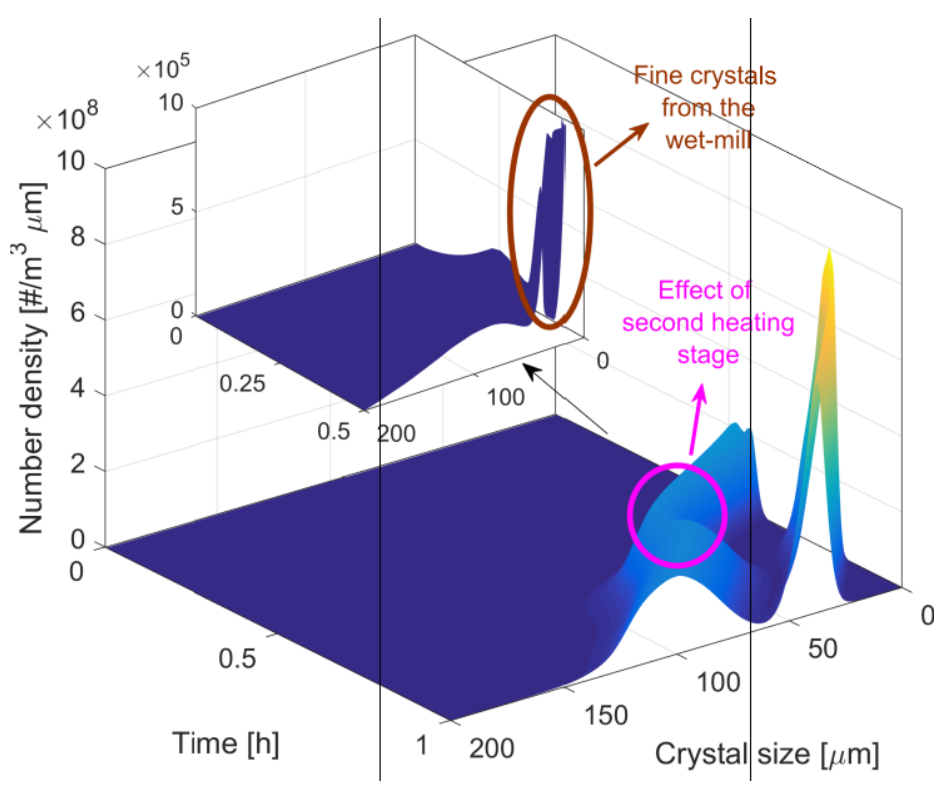

c)

Figure 7. Third optimization study - bimodal target CSD: a) optimal trajectories of temperature, stirring rate and circulation flowrate; b) normalized target and product CSDs; and c) 3D representation of the CSD evolution in the crystallizer during the process. 
Although the simulation and optimization results are promising, a potential practical application may require the following control structures:

Concentration control to avoid the dissolution of fines or growth in in the wet mill, since in this study it was assumed that in the wet mill only attrition occurs. If the solution in the wet mill would remain supersaturated and growth would occur, the wet-mill may simply break the growing crystals down to the minimally achievable size -thus supersaturation would be depleted and the system would generate more secondary nuclei.

Since the dynamic seeding is sensitive to the seed quantity, the system is expected to be sensitive to the seed addition rate. If in the wet mill, for the aforementioned reasons, the crystal number differs from the calculated number, the seed recipe should be recalculated - in real time. This leads directly to the need of applying model based feedback control strategies, coupled with state estimator to detect efficiently the deviations from the optimal trajectory.

It is also important to note that internal mill (immersion mill) cannot deliver the same results due to the lack of recirculation stream. These case studies demonstrate the significant improvement in the achievable product CSD space, by applying novel of model-based optimization and qualityby-control $(\mathrm{QbC})$ approaches for the integrated crystallization-wet mill process. The model and methodology presented here provides a systematic process intensification framework for the design and operation of integrated crystallization-wet mill systems.

\section{Conclusions}

In this paper a theoretical analysis of an integrated batch crystallizer-external wet mill system was presented and discussed. Primary nucleation, crystal growth and dissolution was assumed to occur in the crystallizer, whereas fragmentation and attrition were the key mechanisms in the wet mill. 
For the model system the crystallization kinetics and physical properties of ortho amino benzoic acid were applied and size dependent breakage, involving the wet-mill hydrodynamics (stirring rate) for the breakage description was considered. The model was solved using the high resolution finite volume method, by using a mass conserving breakage description. For improved simulation time a highly efficient parallelized implementation using graphical processing units was developed.

The optimization results revealed that the system is suitable for the production of various size crystals, with narrow CSD. Bimodal CSD has also been achieved. The fact that the pure crystallizer completely failed to produce the target CSDs indicates that the application of external wet mill increased significantly the overall system flexibility. One major advantage of this system discovered through the dynamic optimization study - is that it does not require seeds, in contrast, the external wet mill can act as an in-situ seed generator and accumulator, which was used to seed adaptively and dynamically the crystallizer through dynamically varied recirculation flowrate.

\section{Corresponding Author}

*zknagy@purdue.edu,z.k.nagy@lboro.ac.uk

\section{Acknowledgements}

The financial support of the International Fine Particle Research Institution is acknowledged gratefully. Funding from the European Research Council under the European Union's Seventh Frame-work Programme (FP7/2007-2013)/ERC grant agreement No. [280106-CrySys] is also acknowledged.

\section{Author Contributions}


The manuscript was written through contributions of all authors. All authors have given approval to the final version of the manuscript. All authors contributed equally.

\section{Notations}

$\tilde{n}$, approximate discrete daughter number density, -; $a_{i}$, constants of solubility equation, $\mathrm{kg} / \mathrm{kg}^{\circ} \mathrm{C}^{-\mathrm{i}}$; $b$, breakage function, $-; B$, nucleation rate function, $\# / \mathrm{m}^{3} \mathrm{~s} ; c$, concentration, $\mathrm{g} / \mathrm{g} ; C$, Courant, or CFL number, -; $d$, discrete crystal size, $\mu \mathrm{m}$; $d_{i m p}$, wet mill impeller diameter, $\mathrm{m} ; F$, circulation flowrate, $\mathrm{mL} / \mathrm{s} ; G$, growth rate function, $\mu \mathrm{m} / \mathrm{s} ; h$, discrete size step size, $\mu \mathrm{m} ; h_{\text {imp }}$, wet mill impeller thickness, $\mathrm{m} ; k$, discrete time step size, $\mathrm{s} ; k_{i}$, rate constant, it depends; $k_{V}$, volume shape factor, -; $L$, crystal size, $\mu \mathrm{m} ; l$, mesh element, -; $l_{\text {imp }}$, wet mill impeller height, $\mathrm{m} ; M$, mesh size, -; $n$, number density function, $\# / \mathrm{m}^{3} \mu \mathrm{m} ; N$, wet mill impeller rotation speed, rot/s; $S$, breakage selection function, -; $T$, temperature, ${ }^{\circ} \mathrm{C} ; t$, time, $\mathrm{s} ; V$, volume, $\mathrm{m}^{3} ; w_{i}$, weighting factors of the multi-pole objective function, -; $x$, crystal size, $\mu \mathrm{m}$;

\section{Subscripts}

0 , initial value; $a$, attrition; $b$, nucleation; $c$, crystallizer; $f$, fragmentation; $g$, crystal growth; $l$, mesh element; $n$, nucleon; $s$, solubility; $w m$, wet mill; $\kappa$, grid cell, breaking crystal, -;

\section{Superscripts}

$b$, nucleation rate exponent; $g$, growth rate exponent; $m$, discrete time moment; $\beta$, breakage selection function size exponent;

\section{Greek letters}

$\mu_{3}$, third moment of the distribution, $\mu \mathrm{m}^{3} / \mathrm{m}^{3} ; \beta$, breakage selection function size exponent, $-; \delta$, Dirac-delta function, -; $\theta$, sharpness (gradient) of the distribution, -; $\vartheta$, volume fraction of crystals population, -; $\kappa$, grid cell, breaking crystal, -; $\lambda$, breaking crystal size, $\mu \mathrm{m} ; \rho$, density, $\mathrm{kg} / \mathrm{m}^{3} ; \sigma$, 
supersaturation ratio, -; $\sigma_{f}$, dispersion of the fragmentation daughter distribution function, -; $\Phi$, Van Leer's flux limiter function, -;

\section{Abbreviations}

CFL, Courant-Friedrichs-Lewy; CPU, central processing unit; CSD, crystal size distribution; CUDA, compute unified device architecture; DNC, direct nucleation control; FVM, finite volume method; GPU, graphical processing unit; HR, high resolution; MPC, model predictive control; MSMPR, mixed suspension mixed product removal crystallizer; oABA, ortho-amino benzoic acid; PBE, population balance equation; PBM, population balance model; SSC, supersaturation control; SSE, sum squared error; QbC, quality by control

\section{References}

(1) Mersmann, A. Crystallization Technology Handbook; Marcel Dekker Inc., New York, Basel, 2001.

(2) Nagy, Z. K.; Braatz, R. D. Annu. Rev. Chem. Biomol. Eng. 2012, 3 (1), 55-75.

(3) Cao, Y.; Acevedo, D.; Nagy, Z. K.; Laird, C. D. Control Eng. Pract. 2017, 69, 1-8.

(4) Nagy, Z. K.; Braatz, R. D. AIChE J. 2003, 49 (7), 1776-1786.

(5) Mesbah, A.; Nagy, Z. K.; Huesman, A. E. M.; Kramer, H. J. M.; Van Den Hof, P. M. J. IEEE Trans. Control Syst. Technol. 2012, 20 (5), 1188-1201.

(6) Shi, D.; El-Farra, N. H.; Li, M.; Mhaskar, P.; Christofides, P. D. In Chemical Engineering Science; 2006; Vol. 61, pp 268-281.

(7) Nagy, Z. K.; Fevotte, G.; Kramer, H.; Simon, L. L. Chem. Eng. Res. Des. 2013, 91 (10), $1903-1922$.

(8) Ma, C. Y.; Wang, X. Z. J. Process Control 2012, 22 (1), 72-81.

(9) Borsos, A.; Szilagyi, B.; Agachi, P. S.; Nagy, Z. K. Org. Process Res. Dev. 2017. 
(10) Vetter, T.; Burcham, C. L.; Doherty, M. F. Chem. Eng. Sci. 2014, 106, 167-180.

(11) Su, Q.; Nagy, Z. K.; Rielly, C. D. Chem. Eng. Process. Process Intensif. 2015, 89, 41-53.

(12) Nagy, Z. K.; Fujiwara, M.; Braatz, R. D. J. Process Control 2008, 18 (9), 856-864.

(13) Szilágyi, B.; Lakatos, B. G. Comput. Chem. Eng. 2017, 98, 180-196.

(14) Nagy, Z. K. Comput. Chem. Eng. 2009, 33 (10), 1685-1691.

(15) Acevedo, D.; Tandy, Y.; Nagy, Z. K. Ind. Eng. Chem. Res. 2015, 54 (7), 2156-2166.

(16) Aamir, E.; Nagy, Z. K.; Rielly, C. D. Chem. Eng. Sci. 2010, 65 (11), 3602-3614.

(17) Klapwijk, A. R.; Simone, E.; Nagy, Z. K.; Wilson, C. C. Cryst. Growth Des. 2016, 16 (8), 4349-4359.

(18) Borsos, A.; Majumder, A.; Nagy, Z. K. Cryst. Growth Des. 2016, 16 (2), 555-568.

(19) Yang, Y.; Song, L.; Gao, T.; Nagy, Z. K. Cryst. Growth Des. 2015, 15 (12), 5879-5885.

(20) Acevedo, D.; Kamaraju, V. K.; Glennon, B.; Nagy, Z. K. Org. Process Res. Dev. 2017, 21 (7), 1069-1079.

(21) Yang, Y.; Song, L.; Zhang, Y.; Nagy, Z. K. Ind. Eng. Chem. Res. 2016, 55 (17), 4987-4996.

(22) Vetter, T. In AIChE Annual Meeting; 2016.

(23) Ramkrishna, D. Population Balances; Elsevier, 2000.

(24) Randolph, A.; Larson, M. Theory of particulate processes, analysis and techniques of continuous crystallization; Academic Press: Salt Lake City, 1988.

(25) Chakraborty, J.; Kumar, S. Chem. Eng. Sci. 2007, 62 (15), 4112-4125.

(26) Sato, K.; Nagai, H.; Hasegawa, K.; Tomori, K.; Kramer, H. J. M.; Jansens, P. J. Chem. Eng. Sci. 2008, 63 (12), 3271-3278.

(27) Szilágyi, B.; Muntean, N.; Barabás, R.; Ponta, O.; Lakatos, B. G. Chem. Eng. Res. Des. 2015, 93, 278-286. 
(28) Lakatos, B. G.; Szilagyi, B. Cryst. Growth Des. 2015, 15 (12), 5726-5737.

(29) Mesbah, A.; Kramer, H. J. M.; Huesman, A. E. M.; Van den Hof, P. M. J. Chem. Eng. Sci. 2009, 64 (20), 4262-4277.

(30) Randolph, A.; Larson, M. Theory of particulate processes; Academic Press: Salt Lake City, 1973.

(31) McGraw, R. Aerosol Science and Technology. 1997, pp 255-265.

(32) Friesen, W. I.; Dabros, T. J. Chem. Phys. 2003, 119 (5), 2825-2839.

(33) Aamir, E.; Nagy, Z. K.; Rielly, C. D.; Kleinert, T.; Judat, B. Ind. Eng. Chem. Res. 2009, 48 (18), 8575-8584.

(34) Kumar, S.; Ramkrishna, D. Chem. Eng. Sci. 1997, 52 (24), 4659-4679.

(35) Gunawan, R.; Fusman, I.; Braatz, R. D. AIChE J. 2004, 50 (11), 2738-2749.

(36) Ulbert, Z.; Lakatos, B. G. Aiche J. 2007, 53 (12), 3089-3107.

(37) Qamar, S.; Ashfaq, A.; Warnecke, G.; Angelov, I.; Elsner, M. P.; Seidel-Morgenstern, A. Comput. Chem. Eng. 2007, 31 (10), 1296-1311.

(38) Gunawan, R.; Fusman, I.; Braatz, R. D. AIChE J. 2008, 54 (6), 1449-1458.

(39) Szilágyi, B.; Nagy, Z. K. Comput. Chem. Eng. 2016.

(40) Majumder, A.; Kariwala, V.; Ansumali, S.; Rajendran, A. Ind. Eng. Chem. Res. 2010, 49 (8), 3862-3872.

(41) Marchisio, D. L.; Vigil, R. D.; Fox, R. O. J. Colloid Interface Sci. 2003, 258 (2), 322-334.

(42) Borsos, Á.; Lakatos, B. G. Chem. Eng. Res. Des. 2014, 92 (6), 1133-1141.

(43) Szilágyi, B.; Agachi, P. Ş.; Lakatos, B. G. Powder Technol. 2015, 283, 152-162.

(44) Szilágyi, B.; Nagy, Z. K. Comput. Chem. Eng. 2016, 91, 167-181.

(45) Saha, J.; Kumar, J.; B??ck, A.; Tsotsas, E. Chem. Eng. Res. Des. 2016, 110, 114-122. 
(46) LeVeque, R. J. Finite volume methods for hyperbolic problems; Cambridge university press, 2002; Vol. 31.

(47) Temmel, E.; Eicke, M.; Lorenz, H.; Seidel-Morgenstern, A. Cryst. Growth Des. 2016, 16 (12), 6756-6768.

(48) Celik, I. B.; Ghia, U.; Roache, P. J.; Freitas, C. J.; Coleman, H.; Raad, P. E. J. Fluids Eng. 2008, $130(7), 78001$.

(49) Mesbah, A.; Nagy, Z. K.; Huesman, A. E. M.; Kramer, H. J. M.; Hof, P. M. J. Van den. IEEE Transactions on Control Systems Technology. 2012, pp 1188-1201.

(50) Nagy, Z. K. Comput. Aided Chem. Eng. 2008, 25, 139-144. 
For Table of Contents Use Only

Botond Szilagy, Zoltan K. Nagy, Population balance modeling and optimization of an integrated batch crystallizer - wet mill system for crystal size distribution control

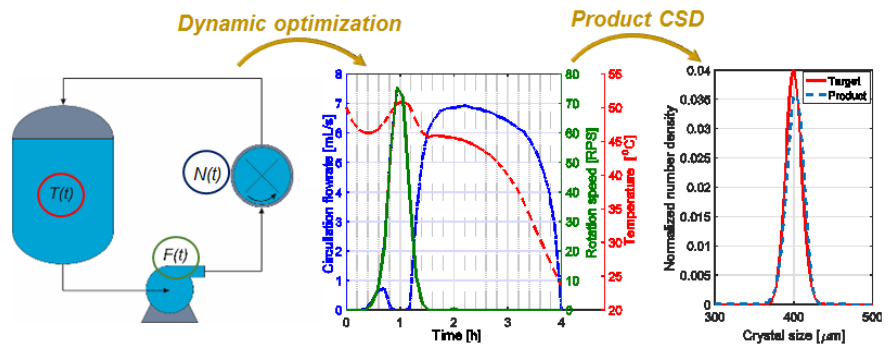

